TECHNOLOGY FEATURE

Putting PTMs on the map

\title{
Understanding how proteins accessorize
}

Proteins can be subjected to a wide variety of targeted post-translational modifications that will considerably modulate their function. Fortunately, several new technologies have emerged to assist in identification and analysis of these modifications, shedding new light on an important layer of proteomic complexity. Caitlin Smith reports.

A growing but extremely important subsector of the field of proteomics involves the study of post-translational modification (PTM), the process by which proteins are enzymatically appended with a wide variety of chemical groups, including lipids, carbohydrate groups and even other proteins. The addition and removal of these groups can dramatically modulate the function of a given protein, and PTMs are known to be involved in the mediation of a staggering variety of processes relating to cell signaling and gene expression, among others.

Unfortunately, the systematic analysis of PTMs is no mean feat. Roland Annan, head of the Proteomics and Biological Mass Spectrometry Group at GlaxoSmithKline, says that one of the biggest obstacles is "the incredible diversity of post-translational modifications and amazing number of different ones that you can find on one gene product. For this reason, I think many individual proteins are their own little proteomes, complex and highly regulated." Although some of the most well-known PTMs include phosphorylation of serine, threonine and tyrosine residues of proteins, there are many other important modifications that also are highly regulated and have a crucial function in cell signaling. Examples of these include acetylation, acylation, glycosylation, methylation, sulfation and ubiquitination, among others. Much of recent research has also focused on histones, where PTMs are thought to be central for dictating the structure of chromatin, affecting cellular proliferation and development.

As daunting as the challenge may seem, however, there are useful tools available

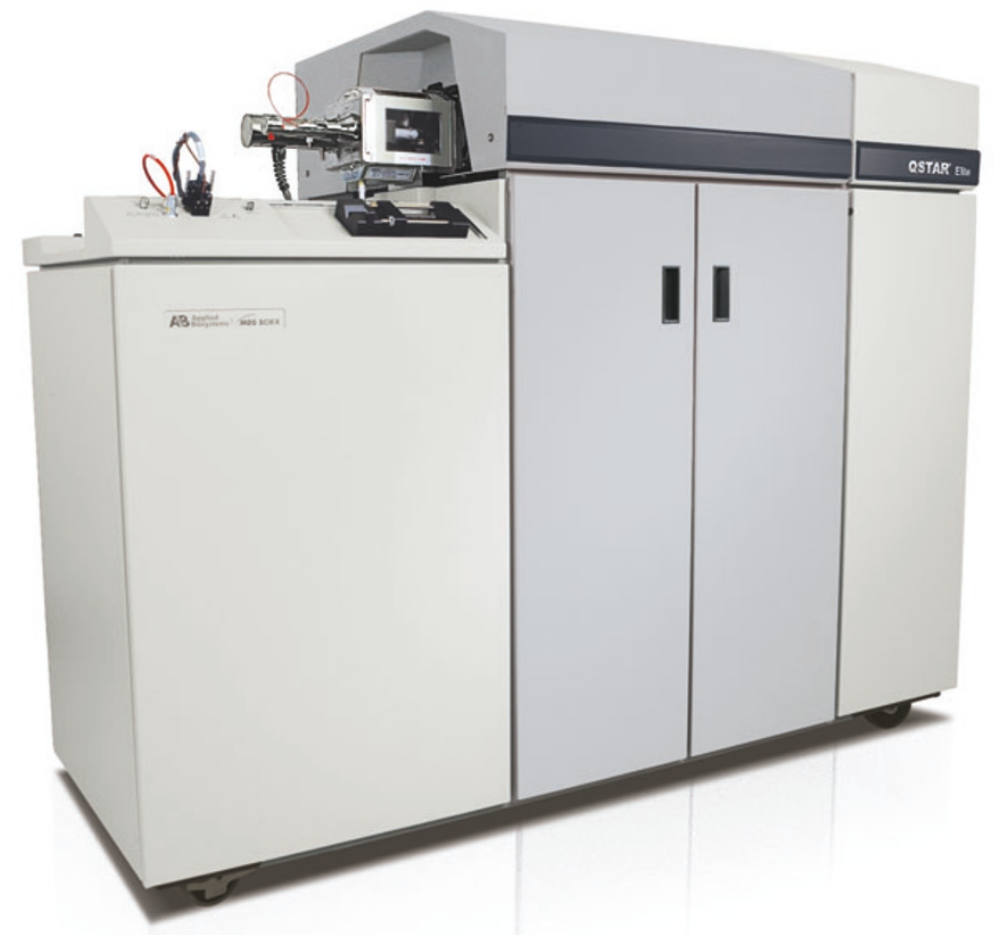

The QSTAR Elite Hybrid LC/MS/MS is among Applied Biosystems' newest solutions for rapid proteomic analysis. (Courtesy of Applied Biosystems.)

to the PTM investigator. "Mass spectrometry has long been a powerful technique to identify post-translational modifications and will continue to be one of the primary methods," says John Yates, a faculty member in cell biology at the Scripps Research Institute. According to Yates, "recent improvements in mass spectrometers (for example, high resolution and high mass accuracy) and new ion dissociation methods have been shown to help identify new types of modifications. Improved resolu- tion and mass accuracy help to differentiate modifications, such as tyrosine phosphorylation and sulfation.” These ongoing developments in mass spectroscopy and associated technologies are now allowing PTM researchers to get more quantitative results and discover more than ever before.

\section{Putting PTMs on the map}

Before mass spectroscopy can be applied to the mapping of PTMs within proteins, 
the protein of interest must be prepared as an appropriate sample for mass spectroscopy analysis. For this 'bottom-up approach' to proteomics, the first step in the preparation process is purification, for which there are a variety of methods. Gel electrophoresis remains a workhorse favorite, with two-dimensional gel electro- spectroscopy preparation. "Two-dimensional electrophoresis remains a method of choice for evidencing post-translational modifications, and also separating modified proteins from unmodified ones, which increases dramatically the probability of finding the modified peptides," says Thierry Rabilloud, of the Laboratory of Cell Bioenergetics, of the French
Atomic Energy Commission. "This is very important when an undirected modification analysis (that is, without any prior hypothesis) is to be performed." Several companies, including Bio-Rad, Topac, Pierce, Sigma-Aldrich and G Biosciences, offer materials for two-dimensional gel electrophoresis. In addition, Nextgen Sciences offers equipment and reagents based on their a2DEoptimizer system.

Another commonly used method is antibody-based affinity purification. Companies are now marketing antibodies directed at all proteins that have a given PTM, such as anti-methyl or anti-phosphotyrosine antibodies. Although there is some question about the effectiveness of these 'pan-PTM' antibodies, they can be useful for a first-pass purification step. Companies offering these antibodies include AbCam, Upstate and Invitrogen.

Once you have a relatively purified sample of protein containing your PTM(s) of interest, the next step involves the chemical or enzymatic degradation of the protein and then separation of the peptides. The latter is usually done by high-performance liquid chromatography (HPLC) and mass spectroscopy (MS) to separate the peptide fragments according to their masses. The mass spectra often show patterns that belie particular PTMs_-for example, multiple mass shifts of $162 \mathrm{kDa}$ for glycosylation sites, and 'satellite' masses of $98 \mathrm{kDa}$ for phosphoserine and phosphothreonine modifications that result from losing phosphoric acid groups.

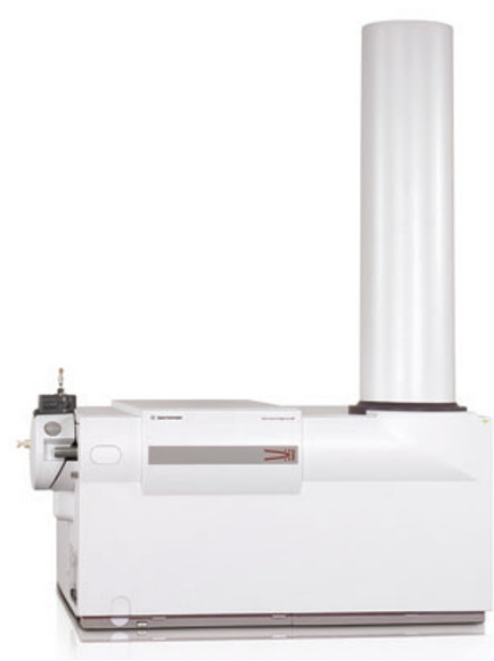

The Agilent 6210 TOF LC/MS system provides a user-friendly platform for integrating fraction collection and mass spectroscopy. (Courtesy of Agilent.) 
Advion Biosciences is trying to speed this process with their TriVersa NanoMate, a system integrating HPLC fraction collection and MS in one platform. In the same vein, Agilent Technologies is aiming for user-friendly performance in their new Agilent 6210 TOF LC/MS mass spectrometer, which can analyze complex mixtures of proteins with greater accuracy and speed of than its predecessor. Not to be outdone, Applied Biosystems recently unveiled its QSTAR Elite LC/MS/MS System, which is billed as fast, accurate and flexible, with the ability to incorporate different types of liquid chromatography workflows.
"The most reliable approach to localize a post-translational modification in the primary structure of a protein or peptide is the recording of tandem MS spectra," says Albert Sickmann, group leader of the Protein Mass Spectrometry \& Functional Proteomics Group at the University of Würzburg. "The interpretation of tandem mass spectra allows the localization of certain mass differences directly to amino acid side chains." In tandem mass spectroscopy, or MS/MS, the fragments are put through another round of MS, in which the peptides themselves are fragmented by collision with an inert gas.

\section{BOX 1 PTM RESEARCH - THE GOOD, THE BAD AND THE UGLY}

Though all PTMs are important from a functional standpoint, they are by no means equal when it comes to ease of study, owing in part to the variable chemical natures of these different PTMs.

Ruedi Aebersold, faculty member at the Institute for Systems Biology at the University of Washington, observes that relatively speaking, all proteins with PTMs are difficult to study, because "modified peptides are rare compared to nonmodified peptides, the modifications may not be stable in a mass spectrometer or during peptide isolation, or modifications may substantially change peptide properties." For example, a lipid attachment may render a peptide relatively insoluble.

"The easy cases are those that are stable, abundant and generate characteristic fragment ion patterns in a mass spectrometer," says Aebersold. "These include oxidized methionine, and phosphotyrosine [modifications]." Marshall of Florida State Univeristy, agrees that the easiest subjects are stable modifications, such as methylation and acetylation. "In particular, we can distinguish trimethylation from acetylation (same mass to the nearest Dalton, but different by $0.036 \mathrm{Da}$ when mass resolving power is high enough)," says Marshall. "A popular target is histones, the positively charged small proteins that package DNA from chromosomes to form particles small enough to fit into cell nuclei. The site(s) and extent of methylation and acetylation determine whether or not particular genes are expressed."

In contrast, it is generally agreed that glycosylation is one of the most difficult PTMs to study, mainly "because of the enormous heterogeneity of glycoforms," says Glaxo Smith Kline Annan. Owing to the vast complexity of glycan structures, Sickmann describes the analysis of glycosylations as "one of the most intriguing challenges in modern proteomics." Other complicating factors, says Sickmann of the University of Würzburg, include "varying amounts of isoforms in between proteins or even single glycosylation sites," as well as transient modifications of the glycan moieties.

Marshall elaborates on this, explaining that one can identify glycosylation sites by ECD, and the branching pattern of the glycan (that is, which sugars are linked to one another) by infrared multiphoton dissociation, "but mass measurement alone can't distinguish different hexoses," says Marshall. "Still, we can distinguish a hexose (for example, galactose) from a pentose (for example, xylose), because the latter has one less carbon; we can identify acetylation of a sugar; and we can identify fucose (methyl versus hydroxyl). However, biochemists often know which sugars are involved, but not how they are interconnected. Although there is a conserved amino acid sequence for sites of $\mathrm{N}$-glycosylation, there is no universal sequence for 0 -glycosylation. Finally, up to dozens of different glycans can occupy the same glycosylation site in a population of glycoproteins." 
Despite the fact that this process can break peptide bonds, some modified amino acids remain intact - another reason that mass spectroscopy techniques are invaluable to PTM researchers, whose subjects can be quite labile (Box 1). "Future developments have to focus on the detection of more labile (for example, glycans, fatty acids) or nearly isobaric (for example, os phosphorylation-sulfation and tri-methylation-acetylation) post-translational modifications," comments Sickmann. Further advances on the horizon, however, will make these labile modifications more amenable to study.

\section{New alternatives for MS}

Advances in mass spectroscopy using the 'top-down' approach to proteomics, in which intact proteins are ionized directly without previous peptide fragmentation, are proving especially fruitful for PTM 을 analysis, yielding greater mass accuracy 을 than ever before. One such approach is ज electron capture dissociation (ECD), a 을 new method that cleaves proteins between

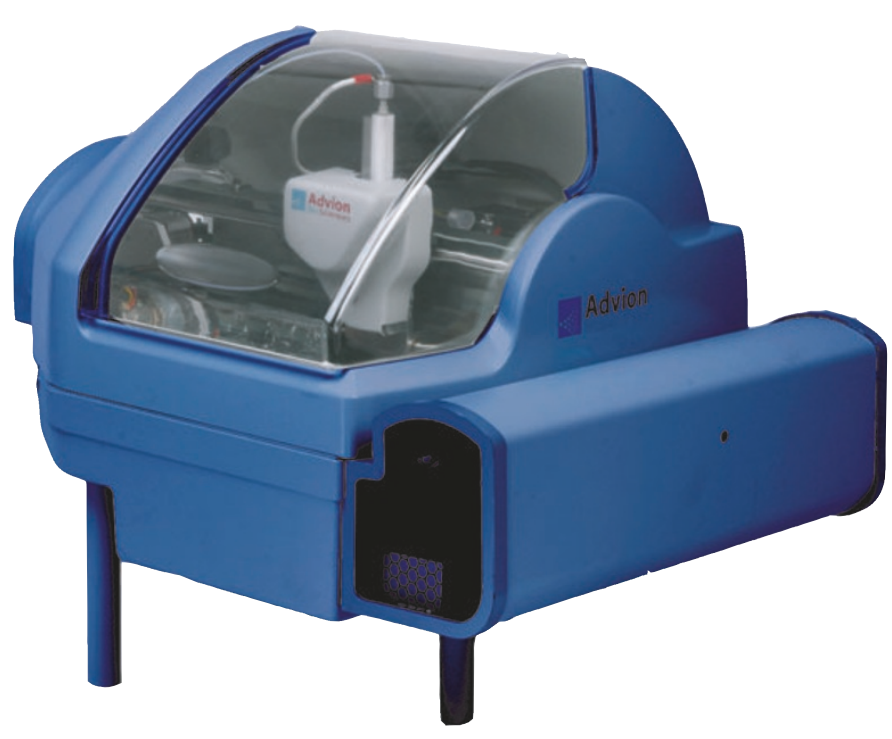

The TriVersa NanoMate, from Advion Biosciences, integrates HPLC fraction collection with MS in a single platform. (Courtesy of Advion Biosciences.)

their backbone amide and alpha carbon. A major advantage of ECD is that labile
PTMs like phosphorylation, $O$-glycosylation, and $\mathrm{N}$-glycosylation remain 


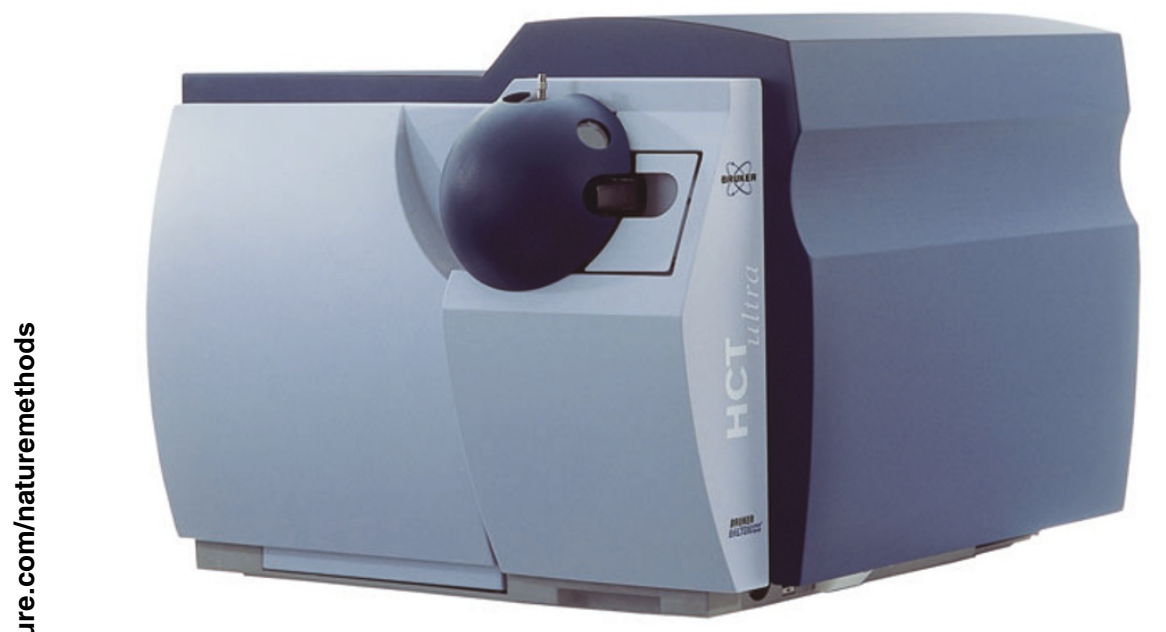

Bruker Daltonics' HCTultra PTM Discovery System uses electron transfer dissociation to fragment proteins while keeping post-translational modifications intact. (Courtesy of Bruker Daltonics.)

attached to the protein backbones, allowing determination of the site and identity of PTMs. Alan Marshall, professor and director of the Ion Cyclotron Resonance Program at Florida State University, describes ECD as "most generally reliable for locating the site of modification without cleaving off the modifying group." This ability to preserve PTMs is making ECD a tool of choice for generating highquality data. Bruker Daltonics recently became the first to offer a commercial instrument for electron transfer dissociation, an alternative method for protein fragmentation that also preserves PTMs. Their HCTultra ion trap facilitates the identification of the types and locations of various PTMs, and is incorporated into their HCTultra PTM Discovery System, which they bill as ideal for researching labile PTMs.

Combining new methods in mass spectroscopy is also a recurring theme in PTM research. For example, Marshall says that combining ECD with other methods, such as infrared multiphoton dissociation or collision-induced dissociation, is especially effective "for determining the branching pattern in glycans [because] ECD cleaves the primary chain, but the two fragments adhere to each other until heated by [infra- 
red radiation].” Roman Zubarev, a professor at Uppsala University, says that a combination of methods is necessary to fragment proteins of interest to the desired degree: "In my opinion, the bottleneck is the absence of high-quality fragmentation techniques.
Collisional activation alone does not deliver full sequence information. A combination of complementary fragmentation techniques will be required, such as collisional activation-electron capture-transfer dissociation or $157 \mathrm{~nm}$ [ultraviolet light] photodisso-

\section{BOX 2 MAKING SENSE OF PTMS-DATABASES AND SEARCH ALGORITHMS}

As in the early days of genomics, researchers in the relatively new field of proteomics find themselves struggling with data management. How do PTM researchers find the information they need? And where do they put new discoveries?

Annan of Glazo Smith Kline says that "most of the search algorithms struggle with post-translational modifications. Many post-translationally modified peptides give less than optimal fragment ion spectra. Many fall off too easily, which makes localizing the site a challenge. This is especially true for ion trap mass spectrometers, which are the most popular types in the field." He notes, however, that recent technical developments in trapping instruments, which use a completely different ion activation mechanism, may soon change this. "Furthermore," says Annan, "since posttranslational modifications are always variable modifications, the search for them can greatly increase the peptide space that an algorithm needs to search through."

Despite the room for improvement, 0ng says that "[software and search algorithms] are invaluable and indispensable. Most of the current packages can make use of highmass-accuracy data to improve the confidence in identification. Some allow first pass searching of the data, setting aside the first set of identifications, then a second pass search that allows a more thorough probing of the data with more variable mass modifications." He thinks that even though current software could be better, "the best way to improve things is really just to use the right instrumentation to generate good data. For instance, using ECD/ETD can provide an almost complete sequence read on modified peptides and even the simplest database search algorithms would be able to handle that data with ease."

Indeed, high-quality MS/MS data are required for the application of ModifiComb, a new software developed by Zubarev's group specifically for the study of PTMs. ModifiComb searches through MS/MS data to find "modified peptides through the similarity of their MS/MS spectra with those of unmodified peptides found in the database or sequenced de novo. ModifiComb gives a semiquantitative picture of all modifications present at once, even if they are novel or unexpected. ModifiComb is capable of mapping hundreds of PTMs simultaneously, including novel modifications, and often the PTMs can be located at a single residue," says Zubarev of Uppsala University.

Sickmann of Würzburg says that for glycan PTMs, "the algorithm-based evaluation of mass spectra needs further improvement, since the evaluation of glycan-derived fragmentation data is still performed predominantly by hand and only few tools and databases (for example, GlycoSuite database or the collection of tools at http://www. glycosciences.de) are presently up to date. Furthermore, the automated analysis of glycopeptide spectra for discrimination between peptide and carbohydrate fragmentation is still at the beginning."

Other tools available include Virtual Expert Mass Spectrometrist (VEMS) and ProSight PTM. VEMS is a program for integrated proteome analysis that is available from Ole Jensen's laboratory webpage (http://yass.sdu.dk). They have developed their own algorithm and software for assignment and quantitation of PTMs in large datasets. The algorithm assigns PTMs by taking advantage of PTM-specific signatures in mass spectra. ProSight PTM, available from Neil Kelleher's lab webpage (https:// prosightptm.scs.uiuc.edu), characterizes PTMs of intact proteins using the 'top down' approach. ProSight PTM can be used with recombinant proteins, proteins in mixtures and protein fragments. 
ciation." Other experts advocate combining 'bottom-up' with 'top-down' approaches for the greatest effectiveness. "In order to be certain," says Shao-En Ong, a scientist at the Broad Institute of MIT and Harvard, "methods combining some fractionation (like liquid chromatography) and very high mass accuracy instruments like the Fourier transform mass spectrometer, combined with rich sequence information from fragmentation experiments (such as ECD fragmentation techniques) are very useful. Instruments that can combine both these elements are therefore certain to be workhorses for PTM, and indeed, proteomic analysis."

\section{Future challenges}

Many proteomics researchers agree that data management is a challenge to their field. Marshall says that big improvements are needed in "the development of a single standardized publicly available platform for data registry, searching, analysis and reporting" for PTMs (Box 2). Andreas Schlosser, a scientist at the Institute of Medical Immunology in Berlin, agrees: "Perhaps the main challenge is to find ways to handle the enormous complexity and amount of data."

Another challenge is the ability to study the PTMs in complex mixtures of proteins. "I think the real difficulty we'll have is how we can study the combination of different PTMs in a single sample," says Ong. "So much of what we know today is based on enrichment from the biological sample. Such protocols are designed to enrich for specific classes of PTM but could potentially miss other modified peptides."

Containing the cost of analysis will also be a major challenge to analyzing PTMs in human samples, according to Zubarev. "The cost of high-quality analysis of one sample is on the order of 100,000 Euro [US $\$ 120,000]$. With such a cost, complete PTM analysis for the human proteome is unrealistically expensive. The cost needs to be reduced by a factor of 100 to put the above task into a realistic budget. Mass spectrometry is progressing very fast, but there is a lot to be done before we even start to approach the possibility of largescale PTM analysis in human samples." He concludes that "high-resolution methods are the only methods that will survive in the long run. The quest for obtaining full sequence information (which is especially important for PTM analysis) has only started."

Caitlin Smith is a freelance writer in Portland, Oregon (caitlin.smith@comcast. net). 


\section{SUPPLIERS GUIDE: COMPANIES OFFERING EQUIPMENT AND REAGENTS FOR PTM STUDIES}

\section{Company}

Abcam

Advion BioSciences

Agilent Technologies

AmProx

AMRESCO, Inc.

Applied Biosystems

Bio-Rad Laboratories, Inc.

Bruker Daltonics

Cambridge Isotope Laboratories, Inc.

Ciphergen Biosystems, Inc.

CTL BIO Services

Dionex Corporation

Extrel

$G$ Biosciences

UVIC - Genome BC Proteomics Centre

Gilson, Inc.

Global Peptide Services, LLC

Invitrogen

JEOL

Kratos Analytical

Matrix Science

NextGen Sciences Ltd.

Palisade Mass Spectrometry

PerkinElmer

Pierce Biotechnology

Proteome Factory AG

ProtTech

Proxeon Biosystems

Shimadzu Corp.

(2) SiDMAP

Sigma-Aldrich

Thermo Electron Corp.

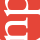

Topac

Upstate

Varian, Inc.

Waters Corp.

\section{Web address}

http://www.abcam.com

http://www.advion.com

http://www.agilent.com

http://www.amprox.com

http://www.amresco-inc.com

http://www.appliedbiosystems.com

http://www.biorad.com

http://www.bdal.de

http://www.isotope.com

http://www.ciphergen.com

http://www.ctlbio.com

http://www.dionex.com

http://www.extrel.com

http://www.gbiosciences.com

http://www.proteincentre.com

http://www.gilson.com

http://www.globalpeptide.com

http://www.invitrogen.com

http://www.jeol.com

http://www.kratos.com

http://www.matrixscience.com

http://www.nextgensciences.com

http://www.palisade-ms.com

http://www.perkinelmer.com

http://www.piercenet.com

http://www.proteomefactory.com

http://www.prottech.com

http://www.proxeon.com

http://www.shimadzu.com

http://www.sidmap.com

http://www.sigma.com

http://www.thermo.com

http://www.topac.com

http://www.upstate.com

http://www.varianinc.com

http://www.waters.com 